\title{
Synthesis, X-ray Structure and Binding Properties of Calix[6]arene-1,4-2,5-Biscrowns
}

\author{
Seung Whan Ko, Byung Ju Ryu, Ki-Min Park, Kwang Hyun No, Jong Seung Kim, and Kye Chun Nam * \\ Department of Chemistry and Institute of Basic Science, Chonnam National University, Gwangiu 500-757, Korea \\ ${ }^{\dagger}$ Department of Chemistry and Research Institute of Natual Seiences, Gyeongsang National Liniversity, Chinju 660-701, Korea \\ ${ }^{+}$Department of Chemistr?, Sookmyung Woman's Liviversity, Seoul 140-7+2, Korea \\ "Deparment of Chemistry, Dankook Universitn: Seonl 140-7/4. Korea \\ Received April 27,2004
}

Key Words : Calix[6]biscrown, X-ray structure, Cesium selectivity

Calixcrowns' are a lamily of macropolycyclic molecules in which the subunits of calixarene and crown ethers are combined through the bridging of phenolic oxygen atoms of the calixarene by polyoxyethylene chains. The lirst member of this family was reported by $\Lambda$ lfieri. ${ }^{2} \Lambda$ s calixcrowns possess well preorganized structures and more rigid binding sites in comparison with calixarenes and crown ethers, they exhibited superior recognition ability toward alkali metal cations. Much attention has been paid to more sophisticated molecules: calix-biscrowns. All possible types of calix|4|arene biscrowns have been synthesized and their recognition propertics toward alkali metal ions have been studied." However, only a few paper conceming the synthesis of calix|6|arene biscrowns has appeared to date. ${ }^{+}$Even for

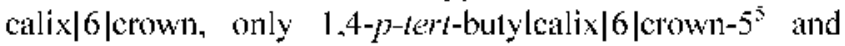
1,4 -calix $|6|$ crown-5 as well as $1,3-p$-tert-butylcalix $[6 \mid$ crown$3^{6}$ have been synthesized.

Cesium-137 is a relatively abundant nuclear fission product and constitutes a major source of heat in nuclear wastes ${ }^{7}$ along with strontium-90. Much effort has been made to the development of improved processes for the removal of ecsium-137 from nuclear wastes. For the purpose of developing cesium selective ion receptors, we prepared two

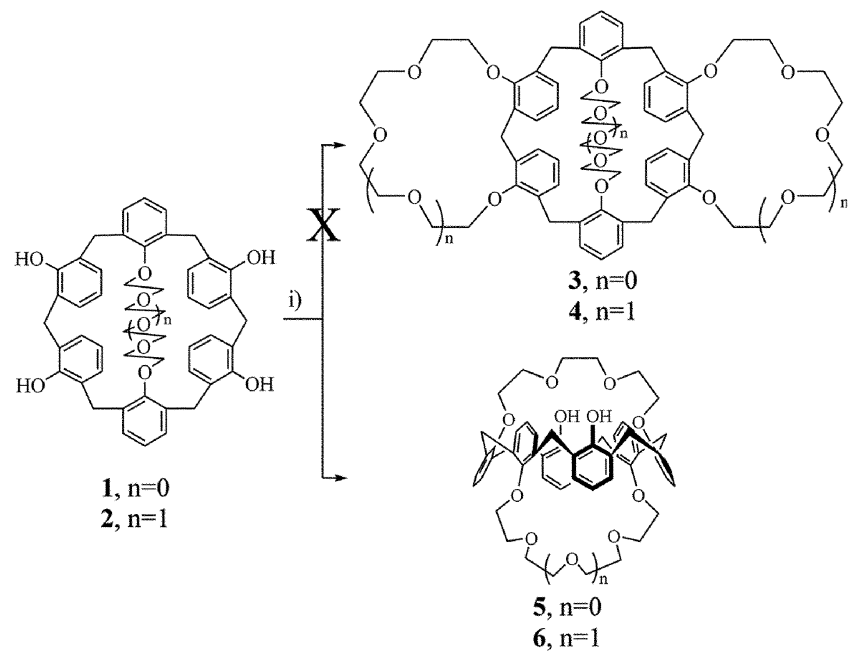

Scheme 1. Synthesis of calix|6|arenc-1.4-2.5-biscrowns. i) $\left(\mathrm{TsOC} \mathrm{H}_{2} \mathrm{CH}_{3} \mathrm{OCH}_{2}\right)_{3} / \mathrm{NaH}$. DMF

\footnotetext{
"Corresponding Author. e-mail: kcnam(achonnam.ac.kr
}

new calix|6|arene biscrowns: calix|6|arene-1,4-2,5-biscrown4 (5) and calix|6|arene-1,4-crown-4-2,5-crown-5 (6) and investigated their X-ray structure and alkali metal ion binding properties.

By treating 1 and $2^{5.6}$ with 2 equivalent of tricthylene glycol ditosylate $\left(\left(\mathrm{TsOCl} \mathrm{I}_{2} \mathrm{CH}_{2} \mathrm{OCl} \mathrm{I}_{2}\right)_{2}\right)$, it was expected to produce the trisbridged calix $[6 \mid c r o w n s ~ 3$ and 4 from the previous results in our lab." But, two bisbridged calix[6]crowns 5 and $6^{(1)}$ were oblained instead in $20 \%$ yicld. It was believed that the long chain of poly ether unit did not allowed to react 2,3-position, rather to bridge 2,5-position of calixarene. Due to the complexity of 'I I NMR spectrum, the conformation of calix|6]arene biscrowns 5 and 6 could nol be deduced. But the X-ray crystallography analysis provide

(a)
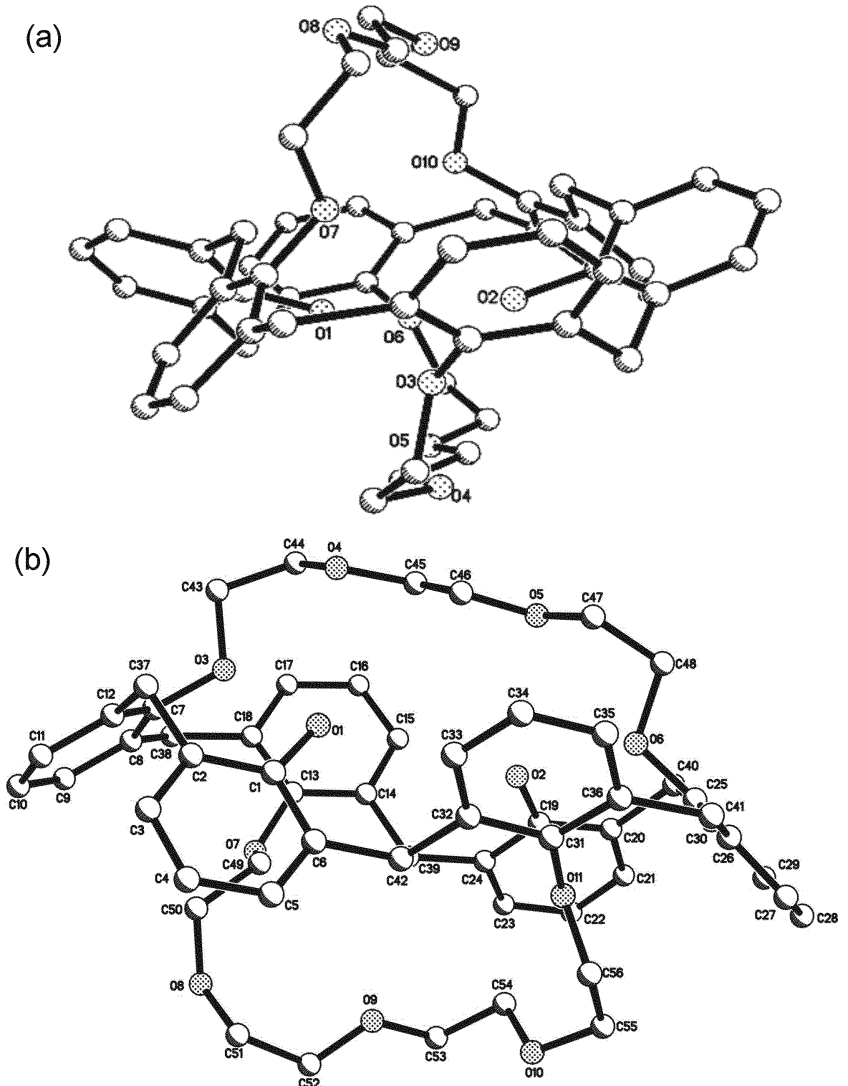

Figure 1. Crystal structure of (a) 5 and (b) 6 . Hydrogen atoms are omitted for clarity. 
Table 1. Crystal data and structure refinement for 5

\begin{tabular}{|c|c|}
\hline Empirical formula & $\mathrm{C}_{54-13} \mathrm{H}_{36,32} \mathrm{ClO}_{10}\left(\mathrm{C}_{5+} \mathrm{H}_{56} \mathrm{O}_{10} \mathrm{l} / 3 \mathrm{CHCl}_{3}\right)$ \\
\hline Crystal stytem & Rhombohedral \\
\hline Space group & $\mathrm{R}-\hat{3}$ \\
\hline Unit cell dimensions & $\begin{array}{l}a=25.0359(17) \AA \alpha=116.1160(10)^{\circ} . \\
b=25.0359(17) \AA \beta=116.1160(10)^{\circ} . \\
c=25.0359(17) \AA \gamma=116.1160(10)^{\circ} .\end{array}$ \\
\hline Refinement method & Full-matrix least-squares on $\mathrm{F}^{*}$ \\
\hline Goodness-of-fit on $\mathrm{F}^{2}$ & 1.748 \\
\hline Final R indices $[I>2$ sigma $(I)]$ & $\mathrm{R} 1=0.1574, \mathrm{wR} 2=0.3814$ \\
\hline $\mathrm{R}$ indices (all data) & $\mathrm{R} 1=0.3526, \mathrm{wR} 2=0.4294$ \\
\hline Largest dift peak and hole & 1.736 and $-0.746 \mathrm{e} \cdot \AA^{-3}$ \\
\hline
\end{tabular}

the alternate conformation.

Figure I showed a X-ray structure of altenate calix[6]arene biscrown 5. Two crown units stayed at the opposite side of calixarene circle. It is believed that this has been the first example of the X-ray structure of alternate conformer of calix[6]arene thus far. Table 1 showed the detail data for $\mathrm{X}$ ray structure 5. A similar X-ray structure of alternate calix[6]arene biscrown 6 was seen in Figure 1. Also two crown units stayed at the opposite side of calixarene circle. Table 2 showed the detail data for X-ray structure 6

The alkali metal binding properties were investigated from two phases extraction experiment where aqueous solutions of the picrate salts are shaken with methylene chloride solutions of ligands. The amount of metal ion was determined from picrate absorption in the UV spectrum. Table 3 showed the percent extraction of metal picrate salts by 5 and 6. The ligand 5 extracted $93 \%$ of the cesium picrate from aqueous solution into the methylene chloride solution of the host, but also $87 \%$ of the rubidium, $61 \%$ of the potassium.

Table 2. Crystal data and structure refinement for 6

\begin{tabular}{ll}
\hline Empirical formula & $\mathrm{C} s \mathrm{H}_{* 4} \mathrm{O}_{\mathrm{L}}$ \\
Crystal system & Monoclinic \\
Space group & $\mathrm{P} 2(\mathrm{I}) / \mathrm{c}$ \\
Unit cell dimensions & $\mathrm{a}=12.3322(\mathrm{I7}) \AA \alpha=90^{\circ}$. \\
& $\mathrm{b}=18.945(3) \AA \beta=97.668(3)^{\circ}$. \\
& $\mathrm{c}=21.445(3) \AA \gamma=90^{\circ}$. \\
Retinement method & Full-matrix least-squares on $\mathrm{F}^{2}$ \\
Goodness-of-tit on $\mathrm{F}^{2}$ & 0.943 \\
Final R indices [I $>2$ sigma(I)] & $\mathrm{RI}=0.0749, w \mathrm{R} 2=0.1878$ \\
R indices (all data) & $\mathrm{RI}=0.1829, w \mathrm{R} 2=0.244 \mathrm{I}$ \\
Largest dift. peak and hole & 0.496 and $-0.277 \mathrm{e} . \AA^{-3}$ \\
\hline
\end{tabular}

Table 3. Percentage extraction (\%) of alkali picrates from water into $\mathrm{CH}_{2} \mathrm{Cl}_{2}$ at $25^{\circ} \mathrm{C}^{a}$

\begin{tabular}{cccccccc}
\hline \multirow{2}{*}{ Ligands } & \multicolumn{7}{c}{ \% Estraction } \\
\cline { 2 - 8 } & $\mathrm{Li}^{-}$ & $\mathrm{Na}^{-}$ & $\mathrm{K}^{-}$ & $\mathrm{Rb}^{-}$ & $\mathrm{Cs}^{-}$ & $\mathrm{NH}_{+}^{+}$ & $\mathrm{Me}_{2} \mathrm{NH}_{2}^{-}$ \\
\hline 5 & 6 & 37 & 61 & 87 & 93 & 5 & 2 \\
6 & 0 & 13 & 28 & 78 & 89 & 3 & 2 \\
\hline
\end{tabular}

"I $\times 10^{-3} \mathrm{M}$ receptor solution in $\mathrm{CH}_{2} \mathrm{Cl}_{\underline{\Sigma}}$ was shaken $(15 \mathrm{hrs})$ with $\mathrm{I} \times 10^{-1}$ $M$ picrate salt solution in $\mathrm{H}_{2} \mathrm{O}$.
$37 \%$ of the sodium and $6 \%$ lithium was extracted. Extraction percentage of cesium salt slightly decreased to $89 \%$ for 6 , potassium and sodium extraction was reduced remarkably as compared to ligand 5 . indicating that the large crown unit could inhibit the effective binding for small metal ions. ISE experiments are in progress using PVC membrane electrodes.

Acknowledgment. This research was supported by Grant (No. KRF-2002-042-C00044) of Cooperative Research Project from Korea Research Foundation.

\section{References and Notes}

1. (a) Gutsche. C. D. Calixarenes: Stoddart. J. F., Ed.: Royal Society of Chemistry: Cambridge. 1989: vol. 1. (b) Vicens. J: Böhmer. V. Calizarene: A Iersatile Class of Aacrocyclic Componds: Kluwer Academic: Dordrecht. 1991. (c) Bohmer. V. Angew: Chem., Int. Ed. Engl. $1995,3+713$.

2. Alfieri. C.: Dradi, E: Poclini. A.: Ungaro, R.: Andreetti, G. D. $J$ Chem. Soc. Chen. Commun 1983, 1073.

3. (a) Mathieu. A.: Asfari. Z: Vicens. T. Tetrahedron Lett. 2002. 43. 1225. (b) Haverlock. T. T.: Mizadeh. S.: Moyer. B. A. J. Ant. Chent. Soc. 2003. 125. 1126. (c) Arduini. A. A. E.: Giorgi. G.: Pochini. A.: Secchi, A. J. Org. Chent 2002, 67.6188. (d) Lee, S. H: Kim. J. Y: Ko. J.; Lee. J. Y.: Kim. J. S. J. Org Chem. 2004. 69. 2902. (e) Talnov. V. S.: Talanova, G. G.: Gorbunova. M. G.: Bartsch. R. A. Tetrahedron Lett. 2002. t3. 1629.

4. (a) Blanda. M. T:: Farmer. D. B.: Brodbelt. T. S.: Gooslby. B. T. J. Ant Chent Soc. 2000. 122. 1486 . (b) Ko. S. W: Yang. Y. S.: Mut1. J. H.: Park, K.: Lee. S. S.: Nam. K. C. Bull Korean Chen. Soc. 2002. 23,1379 .

5. Casnati. A.: Jacpozzi. P.: Pochini. A.: Ugozzoli. F Cacciapaglia. R.: Mandolini. L.: Utigaro. R. Tetrahedron 1995. 51. 591.

6. Chent. Y: Li. T.: Xin. J:: Zhong. Z.: Gong. S.: Lu. X. Simh. Conmmin 1999. 29.705

7. Nucl. Waste News 1993. 13.453.

8. (a) Enest, M. V: Bibler. J. P: Whitley, R. D: Linda Wang, N. H. Ind Eng. Chem. Ren 1997, 36. 2775. (b) Mimura. H: Lehto. J.: Harjula. R. J. Nic. Sci. Tech. 1997.34.607.

9. Ko. S. W.: Lee. S. H.: Park. K.: Lee. S. S.: Nam. K. C. Supromol. Chent 2003. 15.117.

10. Some representative spectroscopic data for calis[6]arene-37.4038.41-bis-crown-4 (5) and calix[6]arene-37,40-crown-4-38,41crown-5 (6) are as follows. 5: $\mathrm{mp} \geqslant 220^{\circ} \mathrm{C}$ dec.: ${ }^{1} \mathrm{H} N \mathrm{NR}\left(\mathrm{CDCl}_{3}\right)$ $\delta 7.38$ (s. 2H. OH). 7.30 (d of d. $2 \mathrm{H}$. ArH. $J=7.38 \mathrm{~Hz}$ ). 7.11 (d. 4H. ArH. $J=7.4 \mathrm{l} \mathrm{Hz}$ ). $7.08-6.93$ (m. $10 \mathrm{H}$. ArH). 6.79 (t. $4 \mathrm{H}$. ArH. $J=7.47 \mathrm{~Hz}$ ). 5.52 (s. $2 \mathrm{H} .-\mathrm{OCH}_{2} \mathrm{CH}_{2} \mathrm{O}-$ ). $4.46-4.38$ (d of d. $4 \mathrm{H} . \mathrm{ArCH}_{2} \mathrm{Ar}_{3} J=15.3 \mathrm{~Hz}$ ). 4.11 (s. $2 \mathrm{H}_{3} \mathrm{ArCH}_{2} \mathrm{Ar}$ ), 3.74-3.69 (m. $\left.2 \mathrm{H} .-\mathrm{OCH}_{2} \mathrm{CH}_{2} \mathrm{O}-\right)_{3}, 3.64-3.56\left(\mathrm{~m} .8 \mathrm{H} .-\mathrm{OCH}_{2} \mathrm{CH}_{2} \mathrm{O}-3\right), 3.41-3.20$ (m, $14 \mathrm{H},-\mathrm{OCH}_{2} \mathrm{CH}_{2} \mathrm{O}$ and $\mathrm{ArCH} \mathrm{Ar}_{2}$. $2.65\left(\mathrm{~m}, 2 \mathrm{H}-\mathrm{OCH}_{2} \mathrm{CH}_{2} \mathrm{O}\right.$ ), $2.40\left(\mathrm{ml} .2 \mathrm{H} .-\mathrm{OCH}_{2} \mathrm{CH}_{2} \mathrm{O}-\right)^{13} \mathrm{C} \mathrm{NMR}\left(\mathrm{CDCl}_{3}\right) \delta 154.46 .153 .32$. 135.16. 133.49. 129.81. 128.69. 127.97. 126.90. 124.21 and 119.25 (Ar). 72.74. 70.62 and $70.27\left(-\mathrm{OCH}_{2}-\right) .36 .10$ and 31.32 (ArCH2Ar). 6: $\mathrm{mp} \rightleftharpoons 202{ }^{\circ} \mathrm{C} \mathrm{dec} .{ }^{1} \mathrm{HNMR}\left(\mathrm{CDCl} 1_{3}\right) \delta 7.55(\mathrm{~s}, 2 \mathrm{H}$. $\mathrm{OH}) .7 .30$ (d of d, $2 \mathrm{H}_{3}$, ArH. $J=7.44 \mathrm{~Hz}$ ). 7.23 ( $\mathrm{d}$ of d. $2 \mathrm{H}_{3}$ ArH. $J$ $=9.18 \mathrm{~Hz}$ ). $7.11-6.98(\mathrm{~m} .6 \mathrm{H}, \mathrm{ArH}), 6.94(\mathrm{~d}$ of d. 2H. ArH $J=$ $8.88 \mathrm{~Hz}$ ). 6.87 (t. $2 \mathrm{H}$. ArH. $J=7.5 \mathrm{~Hz}$ ). 6.75 (t. $2 \mathrm{H}$. ArH. $J=7.44$ Hz). 6.52 (d. $2 \mathrm{H}$. ArH. $J=6.72 \mathrm{~Hz}$ ). 4.49 (d. $2 \mathrm{H}$. ArCH $\mathrm{Ar}_{2} \mathrm{Ar} . J=$ $16.9 \mathrm{~Hz}) .4 .19-4.08\left(\mathrm{~m} .6 \mathrm{H} . \mathrm{ArCH}_{2} \mathrm{Ar}\right.$ and $\left.-\mathrm{OCH}_{2} \mathrm{CH}_{2} \mathrm{O}-\mathrm{-}\right) .3 .84(\mathrm{t}$. 2H. $\left.-\mathrm{OCH}_{2} \mathrm{CH}_{2} \mathrm{O}-\right) .3 .71-3.57\left(\mathrm{~m} .9 \mathrm{H}_{3}-\mathrm{OCH}_{2} \mathrm{CH}_{2} \mathrm{O}\right.$ ), 3.49 (d. $\left.1 \mathrm{H} .-\mathrm{OCH}_{2} \mathrm{CH}_{2} \mathrm{O}-J=5.55 \mathrm{~Hz}\right), 3.4 \mathrm{l}-3.05\left(\mathrm{~m}, 14 \mathrm{H},-\mathrm{OCH}_{2} \mathrm{CH}_{2} \mathrm{O}-\right.$ and $\mathrm{ArCH}_{2} \mathrm{Ar}$ ). 2.89 (m. 2H. $-\mathrm{OCH}_{2} \mathrm{CH}_{2} \mathrm{O}-2.27$ (m. $2 \mathrm{H}$. $\left.-\mathrm{OCH}_{2} \mathrm{CH}_{2} \mathrm{O}-\right) .2 .15$ (m. $2 \mathrm{H} .-\mathrm{OCH}_{2} \mathrm{CH}_{2} \mathrm{O}-$ ) ${ }^{13} \mathrm{C} \mathrm{NMR}\left(\mathrm{CDCl}_{3}\right) \delta$ 156.42. 152.92. 134.65. 133.96. 133.62. 130.23. 129.74. 126.60. 124.00. 123.86. 123.47 and 99.82 (Ar). 72.72. 70.85. 70.06 and $57.67\left(-\mathrm{OCH}_{2-}\right), 31.77$ and $31.55\left(\mathrm{ArCH}_{2} \mathrm{Ar}\right)$. 